\title{
Tutorial: Programmable Integrated Photonics
}

\author{
Wim Bogaerts, Xiangfeng Chen, Iman Zand, Mi Wang \\ Hong Deng, Lukas Van Iseghem, Abdul Rahim, Umar Khan
}

Ghent University - IMEC, Department of Information Technology,

Photonics Research Group, Ghent, Belgium wim.bogaerts@ugent.be

Abstract Programmable photonic circuits are optical chips whose functionality can be (re)configured through electronics and software. This programmability enables new functionality, and opens the path to general-purpose photonic chips, similar to electronic FPGAs. This tutorial discusses the operational principles and technology stack for these chips.

\section{Introduction}

The relatively young field of programmable integrated photonics has been enabled by the rapid growth of photonic integrated circuit (PIC) technology, and in particular silicon photonics, which makes it possible to build large-scale optical circuits using the manufacturing technology for CMOS electronics. With this large-scale one can replace optimized custom circuit designs with electrically controlled tunable circuits to compensate for the fabrication variability. Then it does not take a major leap to start thinking about photonic circuits that can be configured through the same driver electronics perform multiple functions, fully controlled through a software layer ${ }^{[1]-[3]}$.

Software-configured PICs come in many flavours, from application-specific chips that can be adjusted or slightly reconfigured to incorporate redundancy, to circuits that can perform a set of distinct functions and even be useful in different application settings. But programmable integrated photonics can really become a game changer with a general-purpose programmable

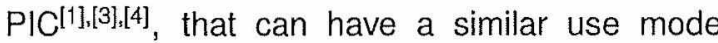
as we see today in programmable electronic chips such as microprocessors, digital signal processors (DSP) and field-programmable gate arrays (FPGA). These have revolutionized the use of electronic circuitry by lowering the threshold for prototyping new electronics-based products: instead of designing and fabricating a new application-specific integrated circuit (ASIC) for every new application, a general-purpose off-theshelf chip is programmed or upgraded.

In this tutorial, we will look at the basic principles and building blocks of these reconfigurable photonic circuits, and the other technological elements that are needed to put them into the hands of programmers to create a use model similar to that of programmable electronics.

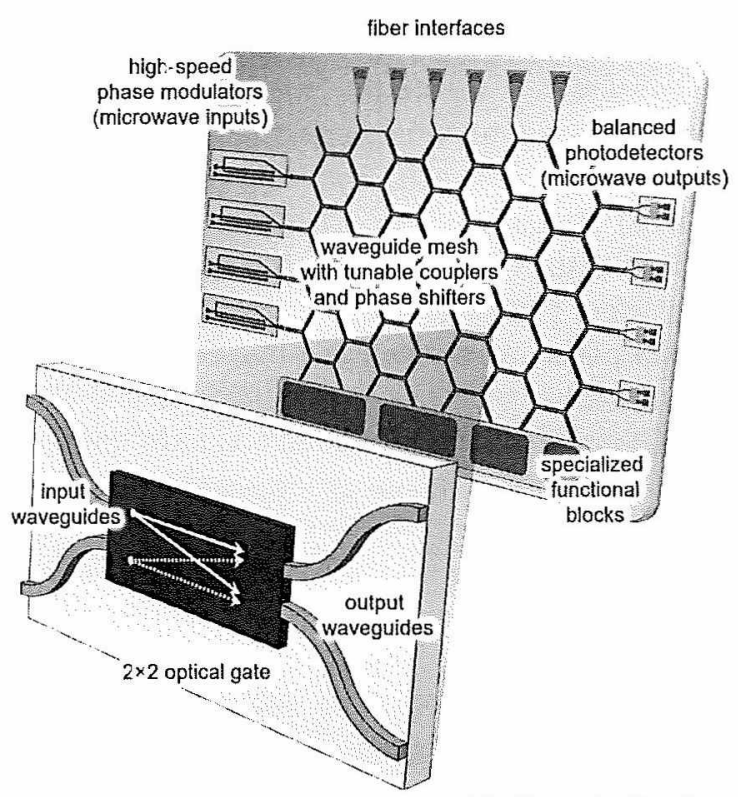

Fig. 1: General-Purpose Programmable Photonic Circuit, consisting of a recirculating waveguide mesh of $2 \times 2$ optical gates connected to fiber ports, modulators, detectors and specialized building blocks.

\section{Programmable Waveguide Circuits}

On a photonic integrated circuit, as schematically illustrated in Fig. 2, lightwaves are processed in different ways: electrical signals are encoded and decoded using modulators and photodetectors, the light is transported and distributed through onchip waveguides, filtered on the basis of wavelength/frequency or polarization, and amplified or transformed in non-linear elements. At the heart of a photonic integrated circuit we find the waveguides which transport the signals between the functional elements. The flow of light within and between the waveguides is governed by optical couplers and phase shifters, and thus waveguides can also be arranged to implement interferometric or resonant wavelength filters.

A programmable photonic circuits generalized these waveguide connections. Instead of designing the circuit as a fixed set of waveguide 
routes, the connectivity is now implemented in a mesh of waveguides that are coupled together with electrically controlled couplers and phase shifters. These form so-called optical $2 \times 2$ gates, which mixes the light of two input waveguides into two output waveguides by controlling the coupling and the differential phase delay between the two arms. When there is no loss inside the gate, the transformation between the complex amplitudes at the input and output ports is unitary ${ }^{[5]}$.

These gates can now be connected together in a waveguide mesh that can have different topologies. We can identify two main classes of meshes: forward-only and recirculating ${ }^{[1]}$. In a forward-only mesh the waveguides are arranged such that light propagates in one direction through the mesh, from a set of input ports to a set of output ports ${ }^{[5]}$. When the mesh has a sufficient number of gates, it can project an arbitrary linear combination of the input ports onto the output ports. This effectively implement a linear matrix-vector product, or a multiply-accumulate (MAC) operation. Such operations form the core of many neural network processes or quantumoptic operations $^{[6]}$.

The recirculating meshes take the connectivity one step further, making it possible for light to traverse the mesh in different directions and even loop back onto itself. The unit cells of such a mesh can be rectangular, triangular or hexagonal, or even combine different unit cells. Depending on the topology, this means that every port can now be used as either an input or an output. It also enables new functionality: the recirculating connectivity makes it possible to implement interferometric filters with optical delay lines and ring resonators. Some recirculating meshes can also be configured to perform the same functionality as the forward-only meshes. All the operations in such a waveguide mesh are linear, so for more advanced functionality, active elements such as modulators, amplifiers, photodetectors or non-linear material need to be introduced.These can be placed either at the edge of the mesh, or incorporated into the unit cells. The optimal strategies for this are still being elaborated, and it is also not yet clear what the best strategy is to scale the size of these waveguide meshes, taking into account optical losses in the $2 \times 2$ gates and the connections to the electrical control layer. This could lead to partitioning and optimization of the mesh: especially in recirculating meshes, it might not be needed to have phase control in ev-
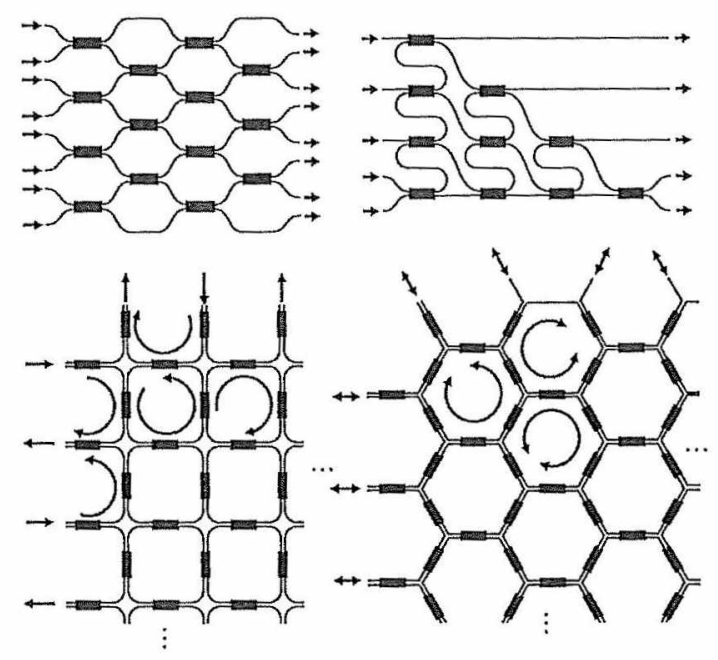

Fig. 2: Different waveguide mesh topologies. Top: Forward-only meshes ${ }^{[5],[6]}$, Bottom: recirculating meshes ${ }^{[7]}$

ery unit cell of the mesh.

Larger meshes impose more stringent requirements on the basic building blocks: waveguides, tunable coupler and optical phase shifters. These need to have a low optical loss, a short optical path length and a low power consumption (because there will be 100 s or 1000s of these on a chip). That means that the the traditional silicon photonics tuning mechanism of heaters becomes less attractive, and alternative actuation mechanisms such as MEMS, phase change materials and liquid crystals present themselves.

\section{More than Photonics}

Even though the optical signals are processed in the photonic circuit, it cannot function without the technologies around it. The manipulation of the optical functions through software requires multiple electronic driver and software layers, as well as packaging technologies to provide the physical interfaces from the photonic chip to the outside world, using fibers for optical signals and highspeed connectors for microwave signals.

Because a programmable photonic chip can have thousands of actively controlled elements, the interface between the photonics and the electronics drivers can be critical constraint, as wirebonding or flip-chipping techniques are limited in the number of pins that can be connected. And that is not counting the need for readout circuits monitoring the on-chip flow of light (which contains a lot of information, in the form of amplitude, phase, wavelength and propagation direction). Programmable photonics is pushing photonicelectronic integration schemes, and monolithic integration or dense 3D stacking could well provide a solution for this in the long term. 
The electronics themselves have different functions. They provide an analog interface to the photonic actuators and monitors, and can even include analog feedback loops, e.g. to minimize simple local cost functions. Such feedback loops can be very fast and power-efficient, and provide a stabilization of the circuit around a given operating point. More complex control and logic to govern the behaviour of the circuit will reside in a higher-up digital electronics layer, implemented in an FPGA, microcontroller or DSP. On top of that, we will then find global configuration and control routines implemented in software.

Programming and controlling such a large photonic circuit is far from trivial, and the work on algorithms is still in its early days. Some configuration schemes, such as a self-adapting beam coupler, can exclusively rely on local feedback loops $^{[5]}$, while high-level multi-routing schemes require heuristic algorithms as the problems scale in a non-polynomial (NP) way. Parasitic effects due to imperfect behaviour of the couplers or phase shifters, or drift of the operating points present another set of challenges to overcome at the algorithmic level.

\section{Applications}

We can classify the implementation and use of programmable photonic circuits into two main catagories: application-specific circuits and general-purpose circuits. And these use cases correspond very well to those we find in electronics: while ASICs almost always deliver superior performance, they are costly to develop, both in terms of time and budget. General-purpose chips, on the other hand, are much more accessible, and dramatically shorten the prototyping phase. even though these chips are usually larger and more power-hungry than ASICs, they can be cost-effective because they are fabricated in larger volumes ${ }^{[4]}$.

One particular function for application-specific programmable PICs is the linear transformation implemented in a forward-only circuit. In the past 5 years we have seen a widespread exploration of such waveguide meshes for use in quantumoptic processors and neural network accelerators $^{[6]}$. These circuits are usually tailored to a specific application to optimize the performance in terms of optical losses and power consumption.

More general-purpose circuits based on recirculating meshes have been less explored. These can be particularly interesting for appli- cations which require reconfigurable connectivity and wavelength filters. Microwave photonics is a field where such functionality is very desirable, and applications include $5 \mathrm{G}$ radio-over-fiber links or low-power radar (e.g. for drones). Other applications include sensor readout circuits (e.g. for fiber Bragg gratings or microring biosensors),

To enable such generic use, the programmable photonic chips should become as accessible as programmable electronics, supporting an ecosystem with developer kits, programming interfaces and classroom material. This could open up the field of integrated photonics to a much wider engineering community, which in turn could unleash new applications based on coherent light.

\section{Conclusions}

The field of programmable photonics is only just starting, and a lot of the technological elements are still in an embryonic phase. Bringing all elements of the technology stack together could create a platform for rapid prototyping of photonic functionality, leading to an entirely new generation of PICs

\section{Acknowledgements}

Part of this work was supported by the European Union grants 780283 (MORPHIC) and 725555 (PhotonicSWARM).

\section{References}

[1] W. Bogaerts, D. Pérez, J. Capmany, et al., "Programmable photonic circuits", Nature, vol. 586, no. 7828 , pp. 207-216, 2020. DOI: 10.1038/s41586-020-2764-0.

[2] D. Pérez, I. Gasulla, P. Das Mahapatra, et al., "Principles, fundamentals, and applications of programmable integrated photonics", Advances in Optics and Photonics, vol. 12 , no. 3, p. 709, 2020. DOl: 10 . 1364/aop . 387155.

[3] J. Capmany and D. Perez, Programmable Integrated Photonics. Oxford University Press, 2020, ISBN: 9780198844402 .

[4] W. Bogaerts and A. Rahim, "Programmable Photonics: An Opportunity for an Accessible Large-Volume PIC Ecosystem", IEEE Journal of Selected Topics in Quantum Electronics, vol. 26, no. 5, p. 1, 2020. DOl: 10.1109/ JSTQE. 2020.2982980.

[5] D. A. B. Miller, "Self-configuring universal linear optical component [Invited]", Photonics Research, vol. 1, no. 1 p. 1, 2013. DOI: 10.1364/prj .1.000001.

[6] N. C. Harris, J. Carolan, D. Bunandar, et al., "Linear programmable nanophotonic processors", Optica, vol. 5, no. 12, pp. 1623-1631, 2018. DOI: 10.1364/optica. 5. 001623.

[7] I. Gasulla, D. Pérez, I. Gasulla, et al., "Programmable multifunctional integrated nanophotonics", Nanophotonics, vol. 7, no. 8, pp. 1351-1371, 2018, ISSN: 21928614. Dol: $10.1515 /$ nanoph-2018-0051. 


\section{Boffi, Pierpaoto}

We2H-6: Mode-group dwision multuplexing for provisioning in SOM networks

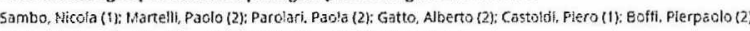

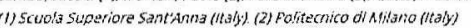

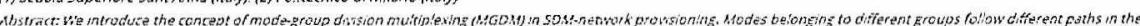

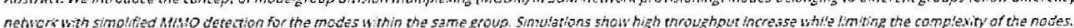

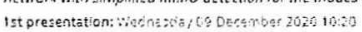

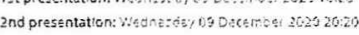

Bogaerts, Vhim

Tu28-2: Silicon Photonic HEMS Add-Drop Fitter

Sattari, Hämed (1): Takabayashi. Alain Yuj) (1): Edinger, Piene (2): Verhayen. Peter (3): Gylfason. Kristnn B. (2): Bogaerts. Wim (4): Quack, Niels (1)

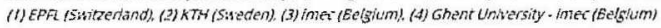

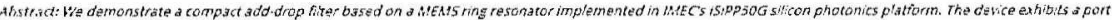

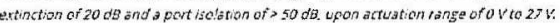

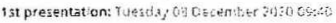

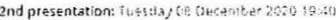

Bogaerts, Yim

Yusa-1: (Tutorial) Programmable Integrated Photonics

Bogaerts, Wim; Chen, Xiangfeng; Zand, Iman; Wang. Mil: Deng. Hong; Vun Iseghem, Lukas: Rahim, Abdul: Khan, Umar

Ghent Unwersity - imec (Belgium)

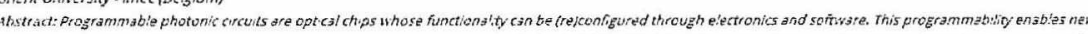

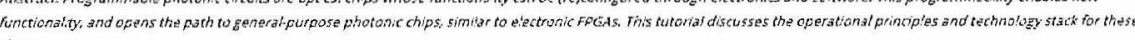

chips.

ist presentation: Tue?

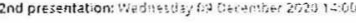

Bogris. Adonts

We 1D-4: Performance and Complextly Evaluation of Recurrent Neural Network Models for Fibre Nonlinear Equalization in Digital Coherent Systems

Delligiannitis. Stavros (1): Mesaritakis, Charis (2): Bogris. Adonis (1)

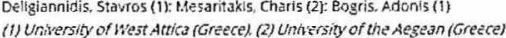

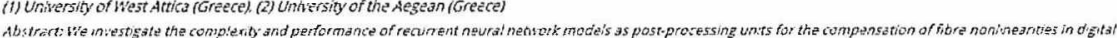

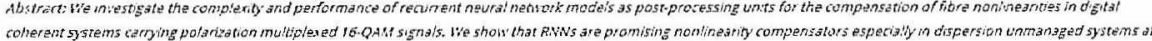

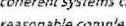

13t pretentiti on: 